\title{
Truncated unscented particle filter for dealing with non-linear and inequality constraints
}

\author{
Miao Yu and Wen-Hua Chen \\ Aeronautical and Automotive Engineering Department \\ Loughborough University,UK, LE11 3TU \\ Email: $\{$ m.yu and w.chen $\} @$ lboro.ac.uk
}

\author{
Jonathon Chambers \\ School of Electronic, Electrical and Systems Engineering \\ Loughborough University,UK, LE11 3TU \\ Email: J.A.Chambers@lboro.ac.uk
}

\begin{abstract}
This paper presents an elegant state estimation method which considers the available non-linear and inequality constraint information. A truncated unscented particle filter method is proposed in this paper. This method applies the particle filtering to cope with non-linear models and non-Gaussian state distribution. Different from other particle filtering schemes, a truncated unscented Kalman filter is applied as the importance function for sampling new particles, in order to incorporate both the measurement and constraint information. Therefore, more effective particles are generated and a better state estimation result is then obtained. The advantages of the proposed truncated unscented particle filter algorithm over the state-of-the-art ones are presented by multiple Monte-Carlo simulations.
\end{abstract}

\section{INTRODUCTION}

State estimation of dynamic stochastic systems is important and receives wide attention in different fields, such as automatic control, signal processing, communication systems, econometrics and so on. The goal of state estimation is to find an estimate of a state using a set of measurements. The dynamics of the state and the relation between the state and measurement are described by a discrete time state model and measurement model respectively.

The state could be estimated from Bayesian inference, from which the conditional probability density function (pdf) of the state conditioned by the measurement is obtained. After deriving the conditional pdf of the state vector by Bayesian inference, the minimum mean square error (MMSE) or maximum a posterior (MAP) estimators [1] could be calculated as the state estimation. As mentioned in [1], if the state and measurement models are both linear and Gaussian, the famous Kalman filter could be applied to obtain an exact solution of the conditional pdf. However, for most real life applications, the linear and Gaussian assumptions do not hold. Different types of algorithms are applied in order to deal with such nonlinear and non-Gaussian models. The classical ones include the variations of the standard Kalman filter (including extended Kalman (EKF) or unscented Kalman filter (UKF)), and particle filter [2].

In some real state estimation problems, the state vector values in stochastic dynamic systems are restricted to a subarea of the state space. This is usually the consequence of some physical restrictions or elicited qualitative knowledge about the systems of interest. For instance, when a vehicle moves on the road, its position is constrained to be within the boundaries of the road and its speed is also generally within the speed limits. Intuitively, because the constraints reduce the variability of the state vector, we can incorporate the constraint-related information into state estimation to achieve a more accurate result.

State estimation with state constraints is in general very challenging and has attracted a number of researchers. Many approaches have been developed to deal with linear and/or equality constraints, which incorporate these constraints into the Kalman filtering framework. The standard ones include reparameterizing and pseudo-measurement approaches [3] and [4], the optimization approach [5] and truncation approaches [6], [7] and [8]; however, under some real scenarios the constraints are both non-linear and inequality. Moreover, the state vector distribution is highly non-Gaussian due to the introduction of these non-linear constraints and the Kalman filtering based methods are then not applicable. In order to more efficiently incorporate the non-linear and inequality constraints, the particle filtering approach is applied and modified to cope with constraints.

Lang et al. in [9] developed a simple and straightforward algorithm using the acceptance-rejection method; particle filtering is applied and only the particles which are within the constraint region are retained. Although simple and straightforward, this method is inefficient and sometimes the probability that a drawn sample meets the constraint is very low. Besides, even if a sample is within the constraint region, it is more likely to be an outlier which has a lower measurement likelihood value. Shao et al. in [10] proposed a novel approach which consists of two stages: First, a set of particle candidates is drawn without consideration of the state constraints; then in stage two, the candidates that do not satisfy the constraints are projected into the feasible area using a series of optimizations. The limitation of this method is that by applying optimizations to force the particle to be within the feasible regions, the resulting particles are no longer representative samples of the posterior distribution of the state vector. So that this method is incorrect from a statistical point of view.

In this work, we propose an elegant truncated unscented particle filtering approach, which is an effective particle filtering method for state estimation and can deal with non-linear and inequality constraints. The proposed truncated unscented particle filter is based on the sequential importance sampling (SIS) method in [2]. And it applies a truncated version of the UKF as the importance function to generate new particles. In this way, both the measurement and constraint information are considered in the sampling procedure and more effective samples are then generated for state estimation. One method 
which is similar to our truncated unscented particle filter is proposed in [11]; however, their method only considers the first two moments of the posterior distribution. This Gaussian approximation leads to less accurate state estimation results.

The outline of this paper is as follows: Section II briefly describes the general problem of state estimation with constraints. The concept of truncated UKF is proposed in Section III and Section IV presents the proposed truncated UPF idea for state estimation. The simulation results are presented in Section V, from which we can see that our proposed method achieves a better performance than the current state-of-the-art ones.

\section{STATE ESTIMATION WITH CONSTRAINTS}

Supposing a stochastic dynamic system with state and measurement models described as:

$$
\begin{aligned}
& \mathbf{x}_{k+1}=f_{k}\left(\mathbf{x}_{k}, \mathbf{u}_{k}\right)+\mathbf{w}_{k} \\
& \mathbf{z}_{k}=h_{k}\left(\mathbf{x}_{k}\right)+\mathbf{v}_{k}
\end{aligned}
$$

where $\mathbf{x}_{k} \in R^{n_{x}}$ represents the state vector, $\mathbf{u}_{k} \in R^{n_{u}}$ represents the control vector and $\mathbf{z}_{k}$ is the measurement vector; $f_{k}(\cdot, \cdot)$ and $h_{k}(\cdot)$ are linear/non-linear functions which are determined by different types of state/measurement models; and $\mathbf{w}_{k}$ and $\mathbf{v}_{k}$ represent the state and measurement noises, which can be described by the pdfs $p\left(\mathbf{w}_{k}\right)$ and $p\left(\mathbf{v}_{k}\right)$. In this work, $\mathbf{w}_{k}$ and $\mathbf{v}_{k}$ are assumed to have Gaussian distributions with $p\left(\mathbf{w}_{k}\right)=N\left(\mathbf{0}, Q_{k}\right)$ and $p\left(\mathbf{v}_{k}\right)=N\left(\mathbf{0}, R_{k}\right)$.

The aim of the state estimation is to estimate the state vector $\mathbf{x}_{k}$ based on the measurement $\mathbf{z}_{k}$ according to the stochastic dynamic system described in (1). From the conditional pdf $p\left(\mathbf{x}_{k} \mid \mathbf{z}_{k}\right)$ derived from Kalman/particle filtering schemes, the minimum mean square error (MMSE) or maximum a posterior (MAP) estimators for $\mathbf{x}_{k}$ could be obtained.

For the real state estimation problem, some other information is applied to refine the distribution of the state vector $\mathbf{x}_{k}$, such as the physical condition which imposes a valid region for the state vector $\mathbf{x}_{k}$. As mentioned in [11], in many scenarios this type of information is represented in a general nonlinear inequality form as:

$$
\mathbf{a}_{k} \leq \mathbf{C}_{k}\left(\mathbf{x}_{k}\right) \leq \mathbf{b}_{k}
$$

where $\mathbf{C}_{k}$ is a mapping function: $R^{n_{x}} \rightarrow R^{n_{c}}$ and $\mathbf{a}_{k}, \mathbf{b}_{k} \in$ $R^{n_{c}}$.

After introducing the constraint in (2), the conditional pdf $p\left(\mathbf{x}_{k} \mid \mathbf{z}_{k}\right)$ is modified to be $p_{C}\left(\mathbf{x}_{k} \mid \mathbf{z}_{k}\right)$ as:

$$
p_{C}\left(\mathbf{x}_{k} \mid \mathbf{z}_{k}\right) \propto \begin{cases}p\left(\mathbf{x}_{k} \mid \mathbf{z}_{k}\right) & \text { if } \mathbf{x}_{k} \in \mathcal{C}_{k} \\ 0 & \text { otherwise }\end{cases}
$$

where $\mathcal{C}_{k}$ is the feasible region which is defined as:

$$
\mathcal{C}_{k}=\left\{\mathbf{x}_{k}:, \mathbf{x}_{k} \in R^{n_{x}}, \mathbf{a}_{k} \leq \mathbf{C}_{k}\left(\mathbf{x}_{k}\right) \leq \mathbf{b}_{k}\right\}
$$

The conditional density function $p_{C}\left(\mathbf{x}_{k} \mid \mathbf{z}_{k}\right)$ could be regarded as a truncation of $p\left(\mathbf{x}_{k} \mid \mathbf{z}_{k}\right)$ by the feasible region $\mathcal{C}_{k}$. It incorporates the constraint information by making the probability values outside the feasible region be zero. With the aid of the truncated conditional pdf $p_{C}\left(\mathbf{x}_{k} \mid \mathbf{z}_{k}\right)$, the uncertainty of the state vector $\mathbf{x}_{k}$ is then reduced and a more accurate state estimation is obtained.

\section{TRUNCATED UNSCENTED KALMAN FILTER}

As mentioned in [12], the truncated UKF is a filtering method which can be applied to estimate the conditional pdf $p_{C}\left(\mathbf{x}_{k} \mid \mathbf{z}_{k}\right)$ considering the constraint information. Truncated UKF is divided into two steps, the first step is to apply the traditional UKF method to estimate the mean and covariance matrix for approximating the conditional pdf $p\left(\mathbf{x}_{k} \mid \mathbf{z}_{k}\right)$. In the second step the Gaussian approximated truncated probability $p_{C}\left(\mathbf{x}_{k} \mid \mathbf{z}_{k}\right)$ is estimated from $p\left(\mathbf{x}_{k} \mid \mathbf{z}_{k}\right)$ to incorporate the constraint information.

\section{A. Unscented Kalman filter}

If the state and measurement models in (1) are linear and Gaussian, the Kalman filter could be applied to calculate the conditional pdf and obtain the state estimation. However, in many situations the linear and Gaussian assumptions do not hold and some variations of the Kalman filter are proposed to deal with the non-linear and non-Gaussian models. One popular variation is the UKF. The UKF is based on the unscented transform (UT), which computes the first two moments of $p\left(\mathbf{x}_{k} \mid \mathbf{z}_{k}\right)$ using a set of $\sigma$-points. Compared with the extended Kalman filter (EKF), it obtains a better estimation if the nonlinearities in state/measurement models are high.

Initially, we have the conditional pdf $p\left(\mathbf{x}_{k-1} \mid \mathbf{z}_{k-1}\right)$ with the mean $\hat{\mathbf{x}}_{k-1 \mid k-1}$ and covariance matrix $P_{k-1 \mid k-1}$ at time $t-1$, a set of $\sigma$-points $\left\{\chi_{i, k-1 \mid k-1}\right\}$ and corresponding weights $\left\{\omega_{i, k-1 \mid k-1}\right\}$ could be calculated as:

$$
\begin{aligned}
\chi_{0, k-1 \mid k-1} & =\hat{\mathbf{x}}_{k-1 \mid k-1}, \quad \omega_{0, k-1 \mid k-1}=\frac{\kappa}{n_{\chi}+\kappa} \\
\chi_{i, k-1 \mid k-1} & =\hat{\mathbf{x}}_{k-1 \mid k-1}+\left(\sqrt{\left(n_{\chi}+\kappa\right) P_{k-1 \mid k-1}}\right)_{i} \\
\omega_{i, k-1 \mid k-1} & =\frac{1}{2\left(n_{\chi}+\kappa\right)} \\
\chi_{n_{\chi}+i, k-1 \mid k-1} & =\hat{\mathbf{x}}_{k-1 \mid k-1}-\left(\sqrt{\left(n_{\chi}+\kappa\right) P_{k-1 \mid k-1}}\right)_{i} \\
\omega_{n_{\chi}+i, k-1 \mid k-1} & =\omega_{i, k-1 \mid k-1}
\end{aligned}
$$

where $n_{\chi}$ and $\kappa$ are preset parameters, $i=1, \ldots, n_{\chi}$ and totally there are $2 n_{\chi}+1 \sigma$-points and $(A)_{i}$ represents the $i$-th column of the matrix $A$.

With the aid of the $\sigma$-points $\left\{\chi_{i, k-1 \mid k-1}\right\}_{i=0, \ldots, 2 n_{\chi}}$ and corresponding weights $\left\{\omega_{i, k-1 \mid k-1}\right\}_{i=0, \ldots, 2 n_{\chi}}$, the mean $\hat{\mathbf{x}}_{k \mid k}$ and covariance matrix $P_{k \mid k}$ of $p\left(\mathbf{x}_{k} \mid \mathbf{z}_{k}\right)$ could be derived by the prediction and correction steps of the UKF algorithm:

\section{Predictions:} as:

The first two moments of $p\left(\mathbf{x}_{k} \mid \mathbf{z}_{k-1}\right)$ could be predicted

$$
\begin{aligned}
& \hat{\mathbf{x}}_{k \mid k-1}=E\left(\mathbf{x}_{k} \mid \mathbf{z}_{k-1}\right) \approx \sum_{i=0}^{2 n_{\chi}} \omega_{i, k-1 \mid k-1} \chi_{i, k \mid k-1} \\
& P_{k \mid k-1}=E\left(\left(\mathbf{x}_{k}-\hat{\mathbf{x}}_{k \mid k-1}\right)\left(\mathbf{x}_{k}-\hat{\mathbf{x}}_{k \mid k-1}\right)^{T} \mid \mathbf{z}_{k-1}\right) \\
& \approx \sum_{i=0}^{2 n_{\chi}} \omega_{i, k-1 \mid k-1}\left(\chi_{i, k \mid k-1}-\hat{\mathbf{x}}_{k \mid k-1}\right)\left(\chi_{i, k \mid k-1}-\hat{\mathbf{x}}_{k \mid k-1}\right)^{T} \\
& +Q_{k-1}
\end{aligned}
$$


where $\chi_{i, k \mid k-1}$ for every $i$ is predicted from the state model as $f\left(\chi_{i, k-1 \mid k-1}, \mathbf{u}_{k}\right)$.

\section{Corrections:}

After receiving the measurement $\mathbf{z}_{k}$, the state prediction results could be updated as:

$$
\begin{aligned}
\hat{\mathbf{x}}_{k \mid k} & =\hat{\mathbf{x}}_{k \mid k-1}+K_{k \mid k}\left(\mathbf{z}_{k}-\hat{\mathbf{z}}_{k \mid k-1}\right) \\
P_{k \mid k} & =P_{k \mid k-1}-K_{k \mid k} P_{z, k \mid k-1} K_{k \mid k}^{T}
\end{aligned}
$$

where $K_{k \mid k}=P_{x z, k \mid k-1}\left(P_{z, k \mid k-1}\right)^{-1}$ is the filter gain and we have the following definitions:

$$
\begin{gathered}
\hat{\mathbf{z}}_{k \mid k-1} \approx \sum_{i=0}^{2 n_{\chi}} \omega_{i} \mathcal{Z}_{i, k \mid k-1} \\
P_{z, k \mid k-1} \approx \sum_{i=0}^{2 n_{\chi}} \omega_{i}\left(\mathcal{Z}_{i, k \mid k-1}-\hat{\mathbf{z}}_{k \mid k-1}\right)\left(\mathcal{Z}_{i, k \mid k-1}-\hat{\mathbf{z}}_{k \mid k-1}\right)^{T}+R_{k} \\
P_{x z, k \mid k-1} \approx \sum_{i=0}^{2 n_{\chi}} \omega_{i}\left(\chi_{i, k \mid k-1}-\hat{\mathbf{x}}_{k \mid k-1}\right)\left(\mathcal{Z}_{i, k \mid k-1}-\hat{\mathbf{z}}_{k \mid k-1}\right)^{T} \\
\mathcal{Z}_{i, k \mid k-1}=h\left(\chi_{i, k \mid k-1}\right)
\end{gathered}
$$

In this way, the mean $\hat{\mathbf{x}}_{k \mid k}$ and covariance $P_{k \mid k}$ of $p\left(\mathbf{x}_{k} \mid \mathbf{z}_{k}\right)$ are updated from the time instance $\mathrm{k}-1$. And $p\left(\mathbf{x}_{k} \mid \mathbf{z}_{k}\right)$ could then be described by these first two moments as a Gaussian distribution denoted as $N\left(\mathbf{x}_{k} \mid, \hat{\mathbf{x}}_{k \mid k}, P_{k \mid k}\right)$.

\section{B. Importance sampling based truncated probability estima- tion}

No constraint information is taken into account for the traditional UKF framework. When the feasible region $\mathcal{C}_{k}$ is considered, according to the definitions in (3) the truncated conditional pdf $p_{C}\left(\mathbf{x}_{k} \mid \mathbf{z}_{k}\right)$ could be calculated as:

$$
p_{C}\left(\mathbf{x}_{k} \mid \mathbf{z}_{k}\right) \propto \begin{cases}\xi_{k}^{-1} p\left(\mathbf{x}_{k} \mid \mathbf{z}_{k}\right) & \text { if } \mathbf{x}_{k} \in \mathcal{C}_{k} \\ 0 & \text { otherwise }\end{cases}
$$

where $\xi_{k}$ is a normalizing constant calculated as:

$$
\xi_{k}=\int_{\mathcal{C}_{k}} p\left(\mathbf{x}_{k} \mid \mathbf{z}_{k}\right) d \mathbf{x}_{k}
$$

As mentioned in [11], a sampling based method could be applied to estimate the mean and covariance of the truncated conditional pdf $p_{C}\left(\mathbf{x}_{k} \mid \mathbf{z}_{k}\right)$, which can then be approximated by a Gaussian distribution. The sampling could be directly applied on $p\left(\mathbf{x}_{k} \mid \mathbf{z}_{k}\right)$ (approximated by $N\left(\mathbf{x}_{k} \mid, \hat{\mathbf{x}}_{k \mid k}, P_{k \mid k}\right)$ from the UKF procedure) and the obtained samples within the constraint region $\mathcal{C}_{k}$ are kept while other samples are discarded; however, we notice that sometimes the probability of obtaining a valid sample is low. The mean and covariance thus can not be estimated accurately by a limited number of samples. In order to solve this problem, we refer to the importance sampling technique. The samples are not obtained directly from $p\left(\mathbf{x}_{k} \mid \mathbf{z}_{k}\right)$, but from another importance function $q\left(\mathbf{x}_{k}\right)$ whose volume should be largely within the constrained region $\mathcal{C}_{k}$. In this work, $q\left(\mathbf{x}_{k}\right)$ is chosen as a Gaussian distribution with the mean being the projection of $\hat{\mathbf{x}}_{k \mid k}$ (the mean estimated by the UKF scheme as in (10)) into the nearest point in the feasible region, and the covariance being $P_{k \mid k}$ which is the same as the one calculated from the UKF scheme.

From a sample set $\left\{\mathbf{x}_{k}^{c, i}\right\}_{i=1, \ldots, N}$ drawn from $q\left(\mathbf{x}_{k}\right)$ in the constraint region $\mathcal{C}_{k}$, the approximate mean $\hat{\mathbf{x}}_{k \mid k}^{c}$ and covariance $P_{k \mid k}^{c}$ of $p_{C}\left(\mathbf{x}_{k} \mid \mathbf{z}_{k}\right)$ could be estimated as:

$$
\begin{gathered}
\hat{\mathbf{x}}_{k \mid k}^{c}=\frac{1}{N} \sum_{i=0}^{N} \mathbf{x}_{k}^{c, i} w_{k}^{c, i} \\
P_{k \mid k}^{c}=\frac{1}{N} \sum_{i=0}^{N}\left(\mathbf{x}_{k}^{c, i}-\hat{\mathbf{x}}_{k \mid k}^{c}\right)\left(\mathbf{x}_{k}^{c, i}-\hat{\mathbf{x}}_{k \mid k}^{c}\right)^{T} w_{k}^{c, i}
\end{gathered}
$$

where $\omega_{k}^{c, i}=N\left(\mathbf{x}_{k}^{c, i} \mid, \hat{\mathbf{x}}_{k \mid k}, P_{k \mid k}\right) / q\left(\mathbf{x}_{k}^{i}\right)$ considering that $p\left(\mathbf{x}_{k} \mid \mathbf{z}_{k}\right)$ is approximated by $N\left(\mathbf{x}_{k} \mid, \hat{\mathbf{x}}_{k \mid k}, P_{k \mid k}\right)$ from the UKF procedure.

By applying a standard unscented Kalman filter followed by an importance sampling based method for truncated conditional probability estimation, the truncated UKF scheme estimates the mean $\hat{\mathbf{x}}_{k \mid k}^{c}$ and covariance $P_{k \mid k}^{c}$ of $p_{C}\left(\mathbf{x}_{k} \mid \mathbf{z}_{k}\right)$, which is approximated by a Gaussian distribution $N_{C}\left(\mathbf{x}_{k} \mid, \hat{\mathbf{x}}_{k \mid k}^{c}, P_{k \mid k}^{c}\right)$. It inherits the advantages of the UKF for coping with highly nonlinear models and incorporates the constraint information in an efficient way.

\section{TRUNCATED UNSCENTED PARTICLE FILTER}

Due to the fact that the distribution is truncated by the constraints, the $p_{C}\left(\mathbf{x}_{k} \mid \mathbf{z}_{k}\right)$ may not be accurately represented by a single Gaussian distribution as estimated from the truncated unscented Kalman filter. In order to represent the conditional pdf in a better way, the particle filtering scheme is applied.

The particle filtering scheme is rooted in Monte-Carlo sampling, which approximates a pdf $p_{C}\left(\mathbf{x}_{k} \mid \mathbf{z}_{k}\right)$ by a set of weights and particles $\left\{\mathbf{x}_{k}^{i}, \omega_{k}^{i}\right\}_{i=1, \ldots, N}$ as:

$$
p_{C}\left(\mathbf{x}_{k} \mid \mathbf{z}_{k}\right) \approx \sum_{i=0}^{N} \omega_{k}^{i} \delta\left(\mathbf{x}_{k}-\mathbf{x}_{k}^{i}\right)
$$

The particle filtering scheme adopts a sequential importance sampling method to estimate $\left\{\mathbf{x}_{k}^{i}, \omega_{k}^{i}\right\}_{i=1, \ldots, N}$ from the weights and samples at $k-1$. An importance function $q\left(\mathbf{x}_{k} \mid \mathbf{x}_{k-1}^{i}, \mathbf{z}_{k}\right)$ is applied to generate the i-th particle $\mathbf{x}_{k}^{i}$ and the corresponding weight $\omega_{i}$ is then updated as in [2]. Unlike the generic particle filter in [2] which generates particles from the importance function merely determined by the state model, the unscented particle filtering (UPF) scheme proposed in [13] applies a UKF to estimate the importance function for generating each particle. The estimated importance function is a local approximation of the optimal importance function given the assumption that the state and measurement models are both linear and Gaussian in a local region nearby each particular particle. By applying the UKF framework to estimate the importance function, the measurement information is incorporated into the particle sampling procedure and generated particles are more likely from the region of high measurement likelihood.

However, for the traditional UPF, the constraint information is not taken into account. In order to make use of the 
constraint information, instead of the UKF, the truncated UKF as mentioned in Section III is applied to obtain the importance function and a corresponding truncated UPF scheme is derived. The procedure of the truncated UPF scheme for estimating $p_{C}\left(\mathbf{x}_{k} \mid \mathbf{z}_{k}\right)$ is outlined as Table I.

Here $p\left(\mathbf{z}_{k} \mid \mathbf{x}_{k}\right)$ and $p\left(\mathbf{x}_{k} \mid \mathbf{x}_{k-1}\right)$ in Table I represent the measurement likelihood function and the state transition function respectively, which are determined by the state and measurement models in (1). $C_{i}$ is the normalization factor considering the constraint on $\mathbf{x}_{k}$, which is estimated as:

$$
C_{i}=\int p\left(\mathbf{x}_{k} \mid \mathbf{x}_{k-1}^{i}\right) \mathbf{C}_{k}\left(\mathbf{x}_{k}\right) d \mathbf{x}_{k}
$$

and it can be estimated from the Monte-Carlo integration as [11], which is similar to the procedure of estimating the mean and covariance of the truncated distribution.

The truncated UPF scheme applies the truncated UKF as the importance function to take both the measurement and constraint information into account in the particle sampling procedure. In this way, a more accurate representation of the truncated conditional density function $p_{C}\left(\mathbf{x}_{k} \mid \mathbf{z}_{k}\right)$ can be obtained.

\section{Simulations}

A vehicle is simulated to move on a bending road section. The boundaries of the road are defined by two arcs centered at the origin of a Cartesian coordinate system with radius of $\mathrm{r} 1=96 \mathrm{~m}$ and $\mathrm{r} 2=100 \mathrm{~m}$, respectively. The vehicle dynamics are described by a white noise acceleration state model as:

$$
\mathbf{x}_{k}=F \cdot \mathbf{x}_{k-1}+G \cdot \mathbf{w}_{k}
$$

where

$$
F=\left[\begin{array}{cccc}
1 & T & 0 & 0 \\
0 & 1 & 0 & 0 \\
0 & 0 & 1 & T \\
0 & 0 & 0 & 1
\end{array}\right], \quad G=\left[\begin{array}{cc}
T^{2} / 2 & 0 \\
T & 0 \\
0 & T^{2} / 2 \\
0 & T
\end{array}\right]
$$

and $\mathbf{x}_{k}=\left[\begin{array}{llll}x_{k} & \dot{x}_{k} & y_{k} & \dot{y}_{k}\end{array}\right]^{T}$. The variables $\left(x_{k}, y_{k}\right)$ represent the position of the vehicle and $\left(\dot{x}_{k}, \dot{y}_{k}\right)$ represents the velocities. $T$ is the sampling interval and assumed to be 1 second. $\mathbf{w}_{k}=\left(w_{k}^{1}, w_{k}^{2}\right)^{T}$ is a $2 \times 1$ vector representing the process noise. Each component of $\mathbf{w}_{k}$ follows a Gaussian distribution with zero mean and a standard deviation 10. Given the road boundary, the state constraints, which are both nonlinear and inequality could be written as:

$$
r_{1} \leq \sqrt{x_{k}^{2}+y_{k}^{2}} \leq r_{2}
$$

The vehicle is tracked by a range and bearing sensor modeled as:

$$
\mathbf{z}_{k}=\left[\begin{array}{c}
\sqrt{x_{k}^{2}+y_{k}^{2}} \\
\arctan \left(\frac{y_{k}}{x_{k}}\right)
\end{array}\right]+\mathbf{v}_{t}
$$

where $\mathbf{v}_{t}$ is a Gaussian noise vector with mean $[0,0]^{T}$ and covariance matrix $R=\left[\begin{array}{cc}5 & 0 \\ 0 & 0.001\end{array}\right]$.

A simulated trajectory according to the state model (22) and the corresponding measurements is plotted in Figure 1.

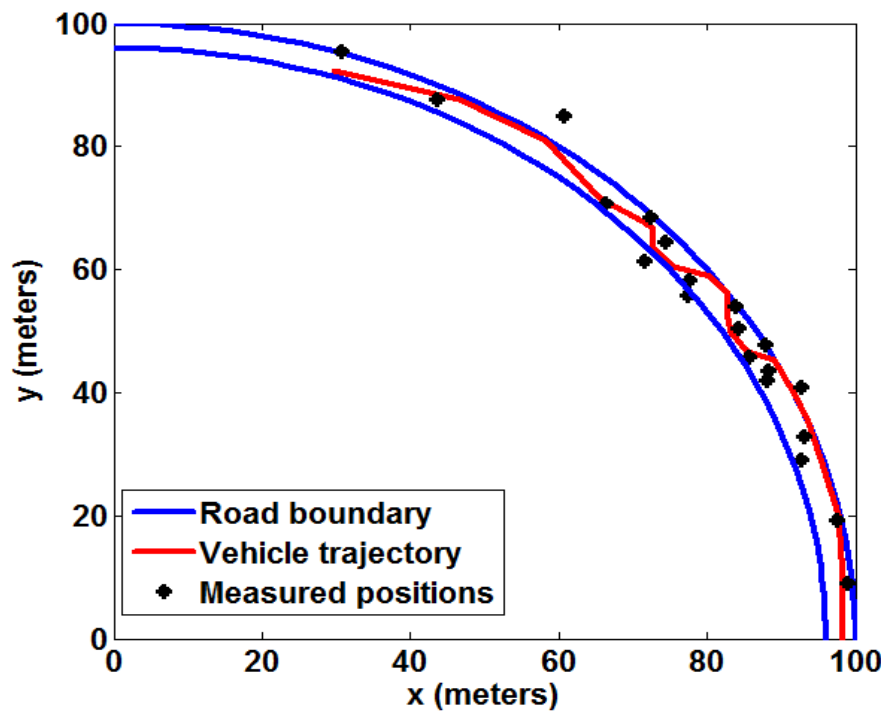

Fig. 1. The simulated trajectory of a vehicle moving on a bend road section and the measured positions.

The incorporating of the state constraint information in (24) could improve the state estimation performance and an example is presented to illustrate it. Figure 2 shows the comparison of the position estimation results by the truncated UPF proposed in this work and the original UPF without considering the constraint information, for each method 100 particles are applied. The mean square errors (MSEs) for these two methods are also calculated. From this figure, we can see that by incorporating the constraint information, the tracking result of truncated UPF is always within the road boundaries and less MSE is obtained.

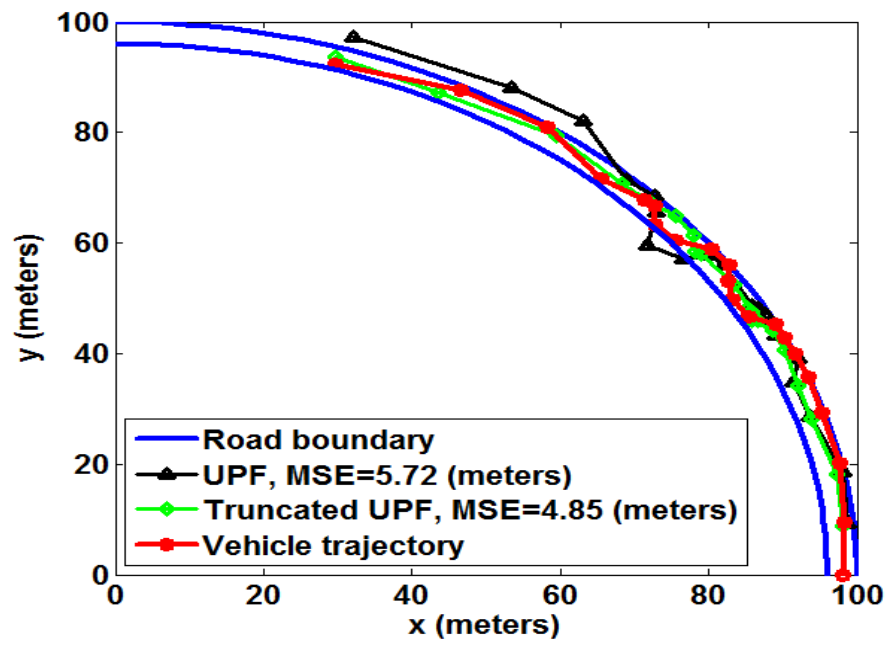

Fig. 2. The comparison results of the T-UPF and standard UPF. Better performance is achieved by T-UPF after incorporating the constraint information.

Next, we compare different methods which could be applied to incorporate the non-linear and inequality constraints. Methods include the accept-rejection method in [9], projection method in [10] and the method proposed in [11]. For a comprehensive analysis, 100 Monte-Carlo simulations are performed to generate the vehicle trajectories and measure- 
TABLE I. The PROCEDURE OF THE TRUNCATED UPF.

Initially, we have a set of particles and weights $\left\{\mathbf{x}_{k-1}^{i}, \omega_{k-1}^{i}\right\}_{i=1, \ldots, N}$ to approximate $p_{C}\left(\mathbf{x}_{k-1} \mid \mathbf{z}_{k-1}\right)$. For each particle $\mathrm{i}$, there is an associated Gaussian distribution $N\left(\mathbf{x}_{k-1} \mid \hat{\mathbf{x}}_{k-1}^{i}, P_{k-1}^{i}\right)$ estimated by the corresponding truncated UKF at time k-1.

\section{Importance sampling:}

For each particle $i$, the truncated UKF in Section III is applied to update $N\left(\mathbf{x}_{k-1} \mid \hat{\mathbf{x}}_{k-1}^{i}, P_{k-1}^{i}\right)$ at $k-1$ to a new distribution $N\left(\mathbf{x}_{k} \mid \hat{\mathbf{x}}_{k}^{i}, P_{k}^{i}\right)$, from which a new particle $\mathbf{x}_{k}^{i}$ is sampled.

\section{Accept and rejection:}

If the obtained sample $\mathbf{x}_{k}^{i}$ is within the constraint region in (2), the sample is accepted; otherwise, it is rejected.

\section{Weight computing:}

The weight corresponding to the accepted particle $\mathbf{x}_{k}^{i}$ is calculated as: $\omega_{k}^{i} \propto \omega_{k-1}^{i} \frac{p\left(\mathbf{z}_{k} \mid \mathbf{x}_{k}^{i}\right) p\left(\mathbf{x}_{k}^{i} \mid \mathbf{x}_{k-1}^{i}\right)}{C_{i} N\left(\mathbf{x}_{k}^{i} \mid \hat{\mathbf{x}}_{k}^{i}, P_{k}^{i}\right)}$

Finally, the weights are normalized to make $\sum_{i=1}^{N} \omega_{k}^{i}=1$ and $p_{C}\left(\mathbf{x}_{k} \mid \mathbf{z}_{k}\right)$ is approximated by the new weights and particles as (20).

ments. Each method with 100 particles is applied to obtain the position estimation results for Monte-Carlo simulations and the corresponding MSEs are calculated. The Mean and Standard deviation(Std) of the 100 MSEs are then estimated for comparison. The results are summarized in Table II, from which we can see that our method achieves the minimum Mean value of the MSEs as well as the smallest Standard deviation compared with other methods.

TABLE II. COMPARISONS OF DIFFERENT ALGORITHMS FOR INCORPORATING THE CONSTRAINT INFORMATION

\begin{tabular}{|c|c|c|c|c|}
\hline & $\begin{array}{c}\text { Accept } \\
\text { rejection [9] }\end{array}$ & Projection [10] & Method in [11] & Proposed \\
\hline $\begin{array}{c}\text { Mean } \\
\text { (meters) }\end{array}$ & 10.89 & 6.92 & 6.76 & 5.40 \\
\hline $\begin{array}{c}\text { Std } \\
\text { (meters) }\end{array}$ & 7.24 & 2.33 & 2.09 & 0.91 \\
\hline
\end{tabular}

\section{CONCLUSIONS}

In this work, we presented a truncated unscented particle filtering scheme to cope with non-linear and inequality constraints. Particle filtering was applied to deal with the non-Gaussian conditional pdf of the state vector due to the introduction of constraints. Unlike the traditional particle filtering methods, the truncated UKF was applied as the importance function from which particles were sampled in the proposed truncated unscented particle filtering scheme. Both the measurement and constraint information were incorporated to obtain a better sampling scheme and a more accurate state estimation result was thus obtained. From multiple Monte Carlo simulations, it was shown that our method achieved a better performance than other state-of-the-art ones.

However, we need to remark that the constraints have different types. Some types of constraints do not impose a feasible region for the state vector as presented in this work, but set the probability likelihoods (in the range of $[0,1]$ ) for the state vector being in different regions. This type of constraint is called a soft constraint [14], in contrast to a hard constraint which makes the probability of being in the feasible region one and the rest zero. Extending the current algorithm to incorporate a soft constraint is the next step for the research.

\section{REFERENCES}

[1] S. Kay, Fundamentals of Statistical Signal Processing Estimation Theory. Prentice Hall PTR, 1993.

[2] B. Ristic, S. Arulampalam, and N. Gordon, "Beyond the Kalman filter: Particle filters for tracking applications," Norwood, MA: Artech House, 2004.

[3] F. Markley, "Attitude error representations for Kalman filtering," Journal of Guidance, Control, and Dynamics, vol. 26, no. 2, pp. 311-317, 2003.

[4] A. Alouani and W. Blair, "Use of a kinematic constraint in tracking constant speed, manouvering targets," IEEE Transactions on Automatic Control, vol. 38, no. 7, pp. 1107-1111, 1993.

[5] C. Agate, R. Wilkerson, and K. Sullivan, "Utilizing negative information to track ground vehicles through move-stop-move cycles," in Proceedings of Signal Processing, Sensor Fusion, and Target Recognition XIII, SPIE, Orlando, FL, USA, 2004.

[6] C. Yang and E. Blasch, "Kalman filtering with nonlinear state constraints," IEEE Transactions on Aerospace and Electronic Systems, vol. 45, no. 1, pp. 70-84, 2009.

[7] B. Teixeira, L. Torres, L. Aguirre, and D. Bernstein, "On unscented Kalman filtering with state interval constraints," Journal of Process Control, vol. 20, no. 1, pp. 45-57, 2010.

[8] S. Julier and J. Laviola, "On Kalman filtering with nonlinear equality constraints," IEEE Transactions on Signal Processing, vol. 55, no. 6, pp. 2774-2784, 2007.

[9] L. Lang, W. Chen, B. Bakshi, P. Goel, and S. Ungarala, "Bayesian estimation via sequential Monte Carlo sampling constrained dynamic systems," Automatica, vol. 43, no. 9, pp. 1615-1622, 2007.

[10] X. Shao, B. Huang, and J. Lee, "Constrained Bayesian state estimation: A comparative study and a new particle filter based approach," Journal of Process Control, vol. 20, no. 2, pp. 143-157, 2010.

[11] O. Straka, J. Dunik, and M. Simandl, "Truncated unscented particle filter," in 2011 American Control Conference, San Francisco, CA, USA, 2011.

[12] O. Straka, J. Dunik, and M. Simandl, "Truncation nonlinear filters for state estimation with nonlinear inequality constraints," Automatica, vol. 48, no. 2, pp. 273-286, 2012.

[13] R. Merve, A. Doucet, N. Freitas, and E. Wan, "The unscented particle filter," Technical Report, Cambridge University Engineering Department, 2000.

[14] F. Papi, M. Podt, Y. Boers, G. Battistello, and M. Ulmke, "On constraints exploitation for particle filtering based target tracking," in 2011 International Conference on Information Fusion, Singapore, 2012. 